\title{
A territorialização como processo de transformação: um relato de experiência
}

\author{
Territorialization as a process of transformation: an experience report
}

La territorialización como proceso de transformación: un informe de experiencia

Raíra Kirlly Cavalcante Bezerra ${ }^{1 *}$, Bruna Passos Vieira ${ }^{1}$, Anne Rafaela de Sousa Ribeiro ${ }^{1}$, Emiliane de Almeida Abreu ${ }^{1}$, Felipe Fabrício Farias da Silva ${ }^{1}$, Rafaele Faustino da Silva de Souza ${ }^{1}$, Deborah Leite de Abreu Souza ${ }^{1}$, Gilvânia Oliveira Ferreira ${ }^{1}$, Izabela de Sousa Paulino'; Maria Vanyele Nogueira Feitosa ${ }^{1}$.

\section{RESUMO}

Objetivo: Descrever estratégias realizadas para o processo de territorialização em saúde capaz de minimizar ou solucionar os principais impasses que dificultam o processo saúde e doença da comunidade. Relato de experiência: Essa pesquisa se caracteriza como um relato de experiência, vivenciada pelos residentes multiprofissionais em Saúde da Família, Saúde Mental e Saúde Coletiva da Escola de Saúde Pública do Ceará, através do processo de territorialização desenvolvida em duas comunidades, localizadas no Sertão Central do Ceará. O reconhecimento do território, inicialmente, se deu por meio de visitas domiciliares dos profissionais residentes, juntamente com o apoio dos agentes comunitários de saúde e, posteriormente realizou-se oficinas como forma de favorecer maior conhecimento dos problemas das comunidades e formular estratégias. Considerações finais: Verificou-se que a territorialização se destaca como uma nova experiência diferenciada de cuidado, dando ênfase na importância do diálogo e da escuta qualificada ao permitir o conhecimento dos fatores que influenciam na qualidade de vida da população. Recomenda-se que esta deve ser utilizada como ferramenta essencial para o planejamento das ações em saúde.

Palavras-chaves: Atenção primária à saúde, Saúde da família, Sistema Único de Saúde.

\section{ABSTRACT}

Objective: To describe strategies for the territorialization process in health, it is able to minimize or resolve the main obstacles that hamper the health-disease process of the community. Experience report: This is an experience report, experienced by the multiprofessional residents of Family Health, Mental Health and Collective Health of the School of Public Health of Ceará, through the territorialization process, developed in two communities, located in the Central Sertão of Ceará. The recognition of the territory the project carries out with activities realized by residents team as visiting homes and with the participation of community health agents, then subsequently realized a series of workshops to foster a greater awareness of the existing community issues nd to develop strategies. Final considerations: It was found that territorialization stands out as a new differentiated care experience, emphasizing the importance of dialogue and qualified listening by allowing the knowledge of factors that influence the quality of life of the population. It is recommended that it should be used as an essential tool for planning health actions.

Keywords: Primary health care, Family health, Unified Health System.

\section{RESUMEN}

Objetivo: Describir estrategias para el proceso de territorialización en salud, capaces de minimizar o resolver los principales impases que obstaculizan el proceso de salud y enfermedad de la comunidad. Informe de experiencia: Esta investigación se caracteriza como un informe de experiencia, vivido por los residentes

${ }^{1}$ Escola de Saúde Pública do Ceará (ESP), Fortaleza - CE. *E-mail: rairakirlly29@gmail.com 
multiprofesionales en Salud Familiar, Salud Mental y Salud Colectiva de la Escuela de Salud Pública de Ceará, a través del proceso de territorialización, desarrollado en el Centro Sertão do Ceará. El reconocimiento del territorio tuvo lugar inicialmente a través de visitas domiciliarias de profesionales residentes, junto con el apoyo de agentes de salud comunitarios, y luego se realizaron talleres como una forma de fomentar un mayor conocimiento de los problemas de las comunidades y para formular estrategias. Consideraciones finales: Se encontró que la territorialización se destaca como una nueva experiencia de atención diferenciada, enfatizando la importancia del diálogo y la escucha calificada al permitir el conocimiento de los factores que influyen en la calidad de vida de la población. Se recomienda que se use como una herramienta esencial para planificar acciones de salud.

Palabras clave: Atención primaria de salud, Salud familiar, Sistema de salud unificado.

\section{INTRODUÇÃO}

A expressão de que todos têm o mesmo direito de acesso às ações e aos serviços de saúde, sem depender da complexidade, do custo e da natureza dos serviços envolvidos, se constitui como caráter universal do Sistema Único de Saúde (SUS). Esse princípio impôs ao Estado a necessidade de um arranjo territorial para organização dos serviços, visto que é impossível prestar assistência á saúde sem antes garantir aos usuários o acesso aos sistemas (PAIM, 1993).

Baseado nesses princípios, o Ministério da Saúde (MS), cria a Estratégia Saúde da Família (ESF), objetivando prestar assistência de qualidade dando ênfase na integralidade e continuidade da assistência, tendo como núcleo fundamental o atendimento à família e o seu espaço social (BRASIL, 1997). Tido como primordial na ESF, o processo de territorialização surge então, sendo capaz de identificar os usuários que estão sobre seus cuidados, realizar acompanhamento contínuo e ações de atenção integral conforme a necessidade de saúde da população local (BRASIL, 2006).

O território, pensado numa perspectiva política e operacional, é caracterizado pela vivência de uma população em um determinado espaço, na qual emerge diversos problemas de saúde, determinantes sociais e outros fatores que influenciam no modo de viver da população. (GONDIM GMM, et al., 2008). A territorialização se caracteriza como uma forma de organização tanto das práticas de saúde, como dos processos de trabalho, a partir da delimitação espacial previamente determinada por meio da caracterização da população e espaço. É parte indispensável para a organização dos serviços na Estratégia Saúde da Família (ESF) (BRASIL, 2006) e já vem sendo utilizada por diversas iniciativas no âmbito do Sistema Único de Saúde (SUS), tais como a Estratégia Saúde da Família, a Vigilância em Saúde Ambiental, a proposta dos municípios/cidades saudáveis e a própria descentralização prevista na Constituição Federal (MONKEN M e BARCELLOS C, 2005).

Caracterizada como uma etapa inicial para o planejamento das ações em uma comunidade, abrangendo enfoques tanto quantitativos quanto qualitativos, o presente relato de experiência, justifica -se pela necessidade de se conhecer a realidade de vida da população, suas potencialidades e dificuldades, para somente assim, partindo dos mais diversos fatores sociais, econômicos e de saúde envolvidos no território, poder traçar estratégias que visem solucionar ou minimizar os problemas observados. $O$ estudo tem como objetivo descrever estratégias realizadas para o processo de territorialização em saúde desenvolvidas por residentes multiprofissionais da Escola de Saúde Pública em um município cearense, capazes de minimizar ou solucionar os principais impasses que dificultam o processo saúde e doença da comunidade.

\section{RELATO DE EXPERIÊNCIA}

Essa pesquisa se caracteriza como um relato de experiência, vivenciada pelos residentes multiprofissionais em Saúde da Família, Saúde Mental e Saúde Coletiva da Escola de Saúde Pública, através do processo de territorialização, desenvolvida nas comunidades da Pompeia e Vila Holanda no município de Quixeramobim. Todos os residentes e profissionais da saúde dos territórios participaram deste processo. Os 
dados foram levantados através de visitas domiciliares, sendo utilizados para esse registro gravador e câmera digital. Esse levantamento ocorreu durante o período de 26 de março a 1 de maio de 2018.

O reconhecimento do território se deu por meio de visitas domiciliares dos profissionais residentes juntamente com o apoio dos agentes comunitários de saúde. Toda a população dos territórios adscritos foram visitados, e as casas que estavam fechadas durante as primeiras visitas, foram visitadas em um outro momento. Durante os encontros, convites de oficinas foram entregues como forma de incentivar, cada vez mais a população, para uma roda de conversa sobre os principais problemas observados durante 0 conhecimento do território, como também, objetivando uma escuta qualificada dos moradores das comunidades sobre os principais impasses que dificultam a moradia em suas localidades.

Após finalizar todas as visitas, em um período médio de trinta dias, deu-se inicio as oficinas, que tinham como objetivo solucionar ou amenizar os principais problemas elencados na territorialização. Realizou-se uma oficina em cada um dos territórios, ambas duraram cerca de duas horas com público médio de 80 pessoas. Elas foram constituídas a partir da abordagem Freireana, que permite um aprendizado rápido e contextualizado na realidade dos educandos.

As atividades realizadas durante as oficinas receberam a denominação "Lavando a roupa suja", sendo desenvolvidas em três etapas. Na primeira etapa, ocorreu uma dinâmica com balões, na qual as pessoas que estavam nos serviços deviam segurar os balões no ar, não os deixando cair no chão. Depois, os residentes convidaram algumas pessoas para saírem da brincadeira, de forma que as pessoas restantes tinham que impedir que os balões caíssem. Ao final da dinâmica, os usuários relataram como foi este momento lúdico para eles, relacionando-o com experiências pessoais, como momentos em família ou amigos, com o contexto social do bairro e com a dinâmica cotidiana de suas vidas.

$\mathrm{Na}$ segunda etapa, os residentes dividiram o público em grupos por setores. Foram divididos grupos dos representantes dos serviços (gestores), profissionais dos serviços de saúde, profissionais dos serviços de assistência social e de educação e usuários. Foram entregues para cada pessoa uma peça de roupa e orientado que elas escrevessem aquilo que acreditavam ser uma problemática que enfrentavam no território, sugerindo que, caso estivessem à vontade, poderiam "pendurar" suas "roupas sujas" no varal, ao final da atividade.

Já na terceira e última etapa da oficina, os residentes entregaram tarjetas amarelas a cada pessoa dos diferentes grupos já divididos, explicando que neste papel deveriam ser escritas algumas soluções para as problemáticas apontadas na etapa anterior. Os residentes explicaram ainda que estas soluções representariam os "raios do sol", no qual as roupas iriam secar.

Através das oficinas e visitas, houve maior aproximação da população com a equipe das Unidades Básicas de Saúde (UBS), e logo foi possível conhecer os espaços e os colaboradores da instituição, assim como realizar os primeiros contatos com o território e com a comunidade, apresentando a Residência e suas finalidades. No tocante às potencialidades dos territórios visitados, destacou-se a presença dos seguintes espaços sociais: tais como, conselho municipal de saúde, praças de lazer, igrejas, como também presença de figuras públicas como rezadeiras, curandeiras e presença política.

Identificou-se também como potencialidades, a importância do acolhimento da equipe técnica nos serviços e dos agentes comunitários de saúde; que com seu conhecimento e disponibilidade, contribuíram muito para a realização das visitas e reconhecimento do território. Foi observado também no território, grande número de adolescentes grávidas, ociosidade de jovens contribuindo para a sua imersão nas drogas ilícitas, condições sanitárias precárias, grande desigualdade social, falta de saneamento básico evidenciado por esgotos a céu aberto, dificuldade de acesso da população à Unidade Básica de Saúde, sendo que estas queixas também foram elencadas pelos participantes.

Dentre as principais dificuldades encontradas durante o processo de territorialização, pode-se elencar: a ausência de transporte para a realização de visitas institucionais nas redes de serviço e nos territórios de atuação; a desarticulação e reduzida participação social durante a execução de algumas oficinas de territorialização e planejamento participativo, principalmente as desenvolvidas com o público da saúde mental; 
a precariedade nas estruturas físicas de alguns equipamentos da rede e a limitação de recursos materiais e de equipamentos. Após o conhecimento dos problemas encontrados na territorialização e desenvolvimento das oficinas, foi possível construir a agenda e a inserção das informações coletadas, suas ações e as estratégias a serem desenvolvidas por cada um dos residentes multiprofissionais com bases nos problemas chaves.

\section{DISCUSSÃO}

O processo de territorialização foi essencial para caracterizar a população e os fatores determinantes que auxiliam e dificultam os processos de trabalho e procura à Unidade Básica de Saúde. Conversar e ouvir a comunidade, usuários, profissionais e representantes dos serviços públicos foi norteador para a elaboração das possíveis intervenções a serem desenvolvidas nas áreas de atuação e nos diferentes núcleos profissionais.

Assim como observado no trabalho de Machado MC, et al. (2012), inicialmente para o processo de realização da territorialização houve uma grande aproximação das equipes das Unidades Básicas de Saúde, o que facilitou o conhecimento dos espaços, território e comunidade.

O conhecer da realidade local torna-se essencial na perspectiva de considerar a saúde em seu sentido ampliado e diversificado. Essa concepção é subsidiada pela renovação do pensamento geográfico de Milton S (1999) que tenta superar uma visão de espaço apenas como algo físico, mas sim como um processo e produto das relações sociais (FARIA RMA, 2009). Autores como Santos AL e Rigotto RM (2010), também reforçam este pensamento de que a territorialização representa um esforço de conhecimento do território e que sempre haverá o que conhecer na dinâmica das territorialidades.

Formação de grupos de fortalecimento de vínculos familiares; melhora no acolhimento nas UBS; realização de ações conjuntas e que proporcionem o diálogo entre os serviços de saúde; prevenção através de palestras educativas; incentivo as denúncias anônimas; orientações quanto ao encaminhamento aos serviços responsáveis Centro de Atenção Psicossocial (CRAS), Centro de Referência Especializado de Assistência Social (CREAS) e Conselho Tutelar; realização de salas de espera nas UBSs; palestras educativas (escolas, creches, etc.); incentivo ao planejamento familiar, pré-natal com as adolescentes grávidas e mobilização da secretaria de infraestrutura, com a participação da comunidade. Estas foram as estratégias elencadas como forma de solução ou minimização dos problemas observados no território, que deverão ser colocadas em prática pelos profissionais de saúde.

Oliveira GN e Furlan PG (2008) definem os processos de territorialização como etapa de suma importância para o conhecimento do território, contribuindo para a construção de "mapas", levando em consideração fatores físicos (barreiras/circulação), socioeconômico, sanitário, demográfico, rede social normativa (listas de equipamentos sociais como escolas, creches, serviços de saúde, instituições religiosas, instituições de apoio social, comércios, etc.), perfil das lideranças comunitárias e organizações associativas, cultural, lazer, etc. Estes fatores estão de acordo com a realidade dos territórios do atual estudo e se assemelham com as estratégias a serem realizadas pelos profissionais de saúde.

A compreensão do território em seu contexto mais amplo, e não apenas como espaço físico, se baseia como um dos grandes fundamentos da territorialização. Araújo GB, et al. (2017), em sua pesquisa, corrobora com o mesmo pensamento ao retratar a importância dos profissionais de saúde na identificação da visão social local acerca do processo saúde-doença; antes mesmo do usuário buscar atenção em uma UBS. E esta identificação da visão social é possível através da inserção do profissional de saúde dentro da comunidade o que muito contribui para facilitação deste contato é o trabalho do Agente Comunitário de Saúde (ACS).

$\mathrm{Na}$ experiência vivenciada, a participação do ACS mostrou-se essencial para um maior conhecimento da comunidade, intensificação do contato entre usuário e a UBS e para facilitação quanto ao processo de construção da identidade do profissional-residente para a população. Por ser um membro da comunidade em que trabalha, o ACS consegue ter uma visão mais ampliada e um conhecimento maior dos impasses que dificultam a convivência e moradia em sua comunidade. O que também pode ser observado no trabalho de 
Nunes MO, et al. (2002), ao intensificar o trabalho do ACS tanto na organização como mediação da comunidade, dando prioridade aos usuários classificados como grupo de risco. BRAND Cl, et al. (2010) em seu estudo, também reforça a importância do trabalho do agente comunitário de saúde, pela constante interação com os valores, satisfações e insatisfações, alegrias, tristezas e linguagem da população e do ambiente em que vivem. Contudo, mesmo com o apoio desses e de tantos outros profissionais de saúde para compreender o território, a população e como ela vive, é importante destacar as dificuldades existentes tanto quanto a resolução dos problemas encontrados nos territórios.

Segundo Faria RMA (2013), a territorialização se dá pela assistência a Atenção Primária à Saúde pelo SUS construindo uma organização nos serviços aos perfis apresentados nos territórios onde essa ação é realizada pelas UBS e a Saúde da Família, acompanhadas no espaço geográfico definido. Porém ao considerarmos que a territorialização preconiza o reconhecimento do território, identificamos que a delimitação de uma área geopolítica e o comprometimento da reorganização das ações em saúde dentro do território, impede o acesso das populações não assistidas por esse território, o que fere o princípio da universalidade do SUS (TETEMANN EC, et al., 2016) e o que dificulta o acesso, principalmente, daqueles mais necessitados, sendo este um obstáculo de difícil resolução por envolver diversos fatores, principalmente os econômicos, sociais e sanitários, mesmo sendo realizado ações e estratégias para minimização dos principais impasses.

\section{CONSIDERAÇÕES FINAIS}

A territorialização abordada neste artigo se configurou como uma forma de organização das práticas de saúde/doença e dos processos de trabalho presentes no território. Foi possível, formular estratégias, objetivando minimizar os principais impasses observados pela equipe de residência e profissionais de saúde nas comunidades adscritas. A territorialização se destaca como uma nova experiência de cuidado, enfatizando o diálogo, a escuta qualificada e a intensificação do vínculo entre os usuários e as equipes das UBS. Assim, recomenda-se a utilização desta como ferramenta de planejamento para as ações das unidades, considerando a territorialização como um processo contínuo e passível de transformações.

\section{REFERÊNCIAS}

1. ARAÚJO GB, et al. Territorialização em saúde como instrumento de formação para estudantes de medicina: relato de experiência. SANARE, 2017; 16 (01): 124-129.

2. BRAND Cl, et al. Satisfações e insatisfações no trabalho do agente comunitário de saúde. Gogitareenferm, $2010 ; 15$ (1):40-47.

3. BRASIL. Secretaria de Assistência à Saúde. Coordenação de Saúde da Comunidade. Saúde da Família: uma estratégia para a reorientação do modelo assistencial. Brasília: Ministério da Saúde, 1997.

4. BRASIL. Secretaria de Atenção à Saúde. Departamento de Atenção Básica. Política nacional de atenção básica / Ministério da Saúde. Brasília: Ministério da Saúde, 2006.

5. FARIA RMA. Territorialização da atenção primária à saúde no Sistema Único de Saúde e a construção de uma perspectiva de adequação dos serviços aos perfis do território. Hygeia, 2013; 9 (16): 131-147.

6. FARIA RM, BORTOLOZZIA. Espaço, território e saúde: contribuições de Milton Santos para o tema da Geografia da Saúde no Brasil. Ra'eGa: O Espaço Geográfico em Análise, Curitiba, 2009; 1(17): 31-41.

7. GONDIM GMM, et al. O território da saúde: a organização do sistema de saúde e a territorialização. In: MIRANDA, Ary Carvalho de et al. (Org.). Território, ambiente e saúde. Rio de Janeiro: Editora Fiocruz, 2008; p.237- 255.

8. JUSTO LG, et al. A territorialização na Atenção Básica: um relato de experiência na formação médica. Interface, comunicação, saúde educação, 2017;21(Supl.1):1345-1354.

9. MACHADO MC, et al. Territorialização como ferramenta para a prática de residentes em saúde da família: um relato de experiência. Rev. Enferm, 2012; 6(11): 2851-2857.

10. MILTON S. A natureza do espaço: espaço e tempo, razão e emoção. 3. ed. São Paulo: Hucitec, 1999.

11. MONKEN M, BARCELLOS C. Vigilância em saúde e território utilizado: possibilidades teóricas e metodológicas. Cadernos de Saúde Pública, 2005; 21(3): 898-906.

12. NUNES MO, et al. O agente comunitário de saúde: construção da identidade desse personagem híbrido e polifônico. Cad Saúde Pública, 2002;18 (6):1639-1646. 
13. OLIVEIRA GN, FURLAN PG. Co-produção de projetos coletivos e diferentes "olhares" sobre o território. In: CAMPOS, G.W. Guerrero A.V.P. Org. Manual de práticas de atenção básica: saúde ampliada e compartilhada. São Paulo: Hucitec, Abrasco; 2008.

14. PAIM JS. A Reforma Sanitária e os Modelos Assistenciais. In: ROUQUAROL, M. Z. (org.). Epidemiologia e Saúde. Rio de Janeiro: MEDSI, 1993, p. 455-466.

15. SANTOS AL, RIGOTTO RM. Território e territorialização: incorporando as relações produção, trabalho, ambiente e saúde na atenção básica à saúde. Trab. Educ. Saúde, 2010; 8(3): 387-406.

16. TETEMANN EC, TRUGILHO SM, SOGAME LCM. Universalidade e Territorialização no SUS: contradições e tensões inerentes. Textos \&Contextos, 2016; 15(2): 356-359. 